\title{
Remanufacturing scheme decision model and application based on reliability evaluation
}

\author{
Xiang Qin 1,a, Zhang Hua ${ }^{1}$ \\ ${ }^{1}$ School of Machinery and Automation, Wuhan University of Science and Technology, 430081, Wuhan
}

Keywords: Remanufacturing scheme, Decision model, Weibull distribution, Three-parameter evaluation, Reliability evaluation.

\begin{abstract}
With the elimination rate of mechanical products is getting higher and higher, it has become a huge problem for manufacturing enterprises to resourcefully dispose the used mechanical products. In order to optimize the resource utilization of the used components, a decision-making model for determining the appropriate remanufacturing plan was established from the perspective of the component reliability based on historical life data. In this study, the three-parameter Weibull distribution function was used to evaluate the component reliability, and the degree of reliability was computed quantitatively. The appropriate remanufacturing plan for the used component was then determined by combining the degree of reliability with the remanufacturing reliability threshold values provided by the manufacturer. The feasibility of the presented model was verified by applying the model to the failure of the bearing on the transfer case. This study can provide technical support for the research and application on remanufacturing scheme decision-making models and active remanufacturing technology of the used components.
\end{abstract}

\section{Introduction}

With the increasing complexity of large-scale machines, the elimination rate of mechanical products is getting higher and higher. Therefore, it has become a huge problem for manufacturing enterprises to resourcefully dispose those used mechanical products. As an important part of the recycling economy, remanufacturing is an industrial process in which the used components are repaired and rebuilt to serve a new purpose, making remanufacturing one of the disposal methods conducive to environmental protection after the end of the product's initial service life [1]. However, due to the different service condition of the used products, even the same type of products, the reliability of its waste components is different [2].

\footnotetext{
${ }^{\mathrm{a}}$ Corresponding author : $249008367 @$ qq.com
} 
Recently, extensive studies have been performed and some achievements were made regarding the remanufacturing scheme decision of the used products or components. Decision models for remanufacturing scheme are usually based on different optimization algorithms [3-5], e.g., recursive algorithms, the case-based reasoning method, and genetic algorithms, where the optimal remanufacturing scheme for the used components is established based on a technological and economic analysis. Comprehensive evaluation models of the remanufacturing decision-making process have been reported in literature for the disposal problem of discarded mechatronic products [6-8], which included economic, technological, resource-related and environmental aspects. Remanufacturing plans can be evaluated both quantitatively and qualitatively. The above studies mainly focused on the aspects of technology, economy, environment and resources, and did not consider an evaluation of the reliability of the used components.

Therefore, a maintenance-mode decision model based on an evaluation of the reliability of the component is proposed in this paper, building on a quantitative analysis of the historical life data of the considered components. The model was then applied to establish a remanufacturing scheme from the perspective of product reliability in terms of the components' lifetime characteristics.

\section{Decision model for the remanufacturing scheme of components}

\subsection{Decision framework}

For standard parts with a low procuring cost and a high remanufacturing cost, they are usually directly replaced after malfunction. Thus, the decision model of the remanufacturing scheme is aimed at non-standard parts and components with a high customization cost. The decision-making framework is illustrated in Figure 1.

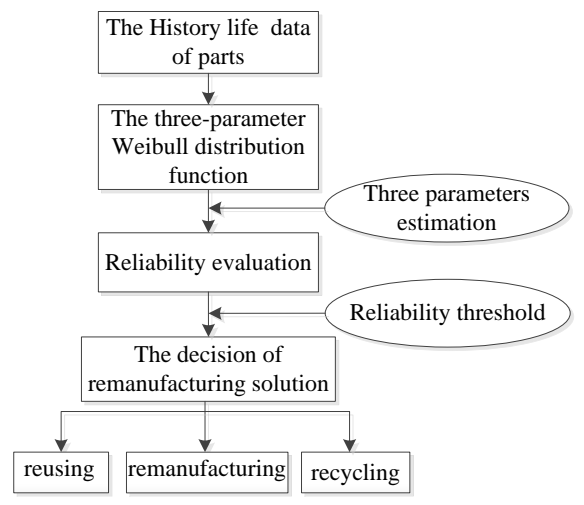

Figure 1. Illustration of the framework of the decision model for the remanufacturing scheme of components.

\subsection{The reliability evaluation of the components}

The Weibull distribution function has been demonstrated to be suitable for describing the lifetime and reliability of mechanical components [9]. There are two basic forms, i.e., a two-parameter distribution and a three-parameter distribution. For the two-parameter Weibull distribution, the calculations required for an estimation of the parameters are simple and flexible, and therefore this method has seen extensive application. However, the parameter estimation is often associated with great errors when the two-parameter Weibull distribution is used to analyze and evaluate the reliability on the basis of empirical data. In contrast, for the evaluation of the service life of mechanical 
components characterized by loss and failure, a higher precision and a more practical reflection of the reliability of the mechanical equipment can be obtained when using the three-parameter Weibull distribution to calculate the lifetime of mechanical components.

\subsubsection{Weibull distribution model}

For mechanical components, the failure rate is related to the actual service time, so the failure rate is a function of the time $(\mathrm{t})$. The Weibull distribution can describe the overall distribution of the probability of failure of a mechanical component over its whole service life.

The failure density function of the three-parameter Weibull distribution is generally expressed as:

$$
f(t)=\frac{\beta}{\eta}\left(\frac{t-t_{0}}{\eta}\right) \exp \left[-\left(\frac{t-t_{0}}{\eta}\right)^{\beta}\right]
$$

The failure distribution function of the Weibull distribution is given by:

$$
F(t)=1-\exp \left[-\left(\frac{t-t_{0}}{\eta}\right)^{\beta}\right]
$$

where $t \geq t_{0}, \beta>0, \eta>0 . t_{0}$ is the position parameter, also called the minimum lifetime. Its value does not affect the shape of the density curve but only shifts the curve. $\beta$ is the shape parameter which determines the shape of the density curve. $\eta$ is the scale parameter, also called the characteristic lifetime. Its value determines the approximate midpoint position of the probability density function.

The component reliability refers to the ability of a component to perform its design functions under stated conditions for a specified period of time. The indexes reflecting the component reliability are mainly the degree of reliability $R(t)$ and the cumulative failure rate $F(t)$, with $R(t)=1-F(t)$, where $R(t)$ is used to reflect the component reliability via the following equation:

$$
R(t)=\exp \left[-\left(\frac{t-t_{0}}{\eta}\right)^{\beta}\right]
$$

To determine the component's degree of reliability, the three parameters $t_{0}, \beta, \eta$ of the Weibull distribution must be estimated first.

\subsubsection{Three-parameter estimation}

Eq. (2) can be transformed into: $1-F(t)=\exp \left[-\left(\frac{t-t_{0}}{\eta}\right)^{\beta}\right]$

Applying the natural logarithm to both sides of the above expression, we obtain:

$\ln \ln \left[\frac{1}{1-F(t)}\right]=\beta\left[\ln t-t_{0}-\ln \eta\right]$

For $Y=\ln \ln \left[\frac{1}{1-F(t)}\right], X=\ln t-t_{0}$ and $A=-\beta \ln \eta$, then Eq. (4) can be rewritten as:

$Y=\beta X+A$

For this linear equation, the least-square estimates of its parameters $\beta$ and $A$ are:

$$
\left\{\begin{array}{l}
\hat{\beta}=\frac{\sum_{i=1}^{n} X_{i} Y_{i}-n \bar{X} \bar{Y}}{\sum_{i=1}^{n} X^{2}-n \bar{X}^{2}} \\
\hat{A}=\bar{Y}-\hat{\beta} \bar{X}
\end{array}\right.
$$


where $\bar{X}=\frac{1}{n} \sum_{i=1}^{n} X_{i}$ and $\bar{Y}=\frac{1}{n} \sum_{i=1}^{n} Y_{i}$.

The correlation coefficient is then given by:

$\rho=\frac{\left[\sum_{i=1}^{n} X_{i} Y_{i}-\frac{\sum_{i=1}^{n} X_{i} \sum_{i=1}^{n} Y_{i}}{n}\right]^{2}}{\left[\sum_{i=1}^{n} X_{i}^{2}-\frac{\bar{X}^{2}}{n}\right]\left[\sum_{i=1}^{n} Y_{i}^{2}-\frac{\bar{Y}^{2}}{n}\right]}$

The position parameter $t_{0}$ is determined first and its initial value $t_{01}$ is the minimum service life according to the experimental data. Then, the three-parameter Weibull distribution is regressed linearly by parameter transformation and the correlation coefficient $\rho_{1}$ is computed. Next, the iterations were started according to $t_{02}=t_{01}-\Delta$ with a step length $\Delta=0.02 t_{01}$. The above process was repeated and the corresponding correlation coefficient $\rho_{\mathrm{i}}$ was calculated for each step. The desired position parameter $t_{0}$ is the $t_{0 i}$ corresponding to the maximal correlation coefficient $\rho_{\mathrm{i}}$.

Next, the mean rank order method [10] was used to determine the corresponding shape parameter $\beta$ and scale parameter $\eta$. For a set of experimental values, some of them are not related to a failure of the component within the data acquisition period. For these values, the mean rank or the approximate middle rank cannot be used. Hence, all of the rank orders had to be estimated according to the failure data and the non-failure data obtained for the component. Thus, the mean rank order was computed and the value of the empirical distribution function was obtained by substituting it into the approximate middle rank equation.

For a sample with non-failure data, the increment formula to compute the mean rank is as follows:

$$
\left\{\begin{array}{l}
\Delta A_{i}=\frac{n+1-A_{i-1}}{n-m+2} \\
A_{i}=A_{i-1}+\Delta A_{i}
\end{array}\right.
$$

where $\mathrm{n}$ is the sample size, $\mathrm{m}$ is the sequence number (from small to large) of the failure or nonfailure time for all components, $A_{i}$ is the mean rank order of the failure component and $A_{i-1}$ is the mean rank order of the previous failure component.

The following expression can be obtained by substituting the above mean rank order $A_{i}$ into the approximate middle rank equation:

$$
F_{n}\left(t_{i}\right)=\frac{A_{i}-0.3}{n+0.4}
$$

The linear regression curve of the Weibull distribution model can be fitted using the least-square parameter estimation method based on the failure time and the computed empirical distribution function. Then, the shape parameter $\beta$ and the scale parameter $\eta$ of the Weibull distribution can be determined. Finally, the component reliability can be computed by plugging the three computed parameters into Eq. (3).

\subsection{Remanufacturing scheme decision for an used parts}

Whether scrap parts have the remaining service life is the precondition for remanufacturing scheme decision-making. The component reliability during the life cycle has a direct influence on the decision for remanufacturing scheme decision-making. The purpose of the decision is to provide a reasonable remanufacturing scheme according to the equipment status and the component reliability. By analyzing the reliability of the discarded components over the life cycle and considering the reliability threshold value of the components provided by the manufacturer, three different remanufacturing schemes are proposed, as shown in Figure 2. 


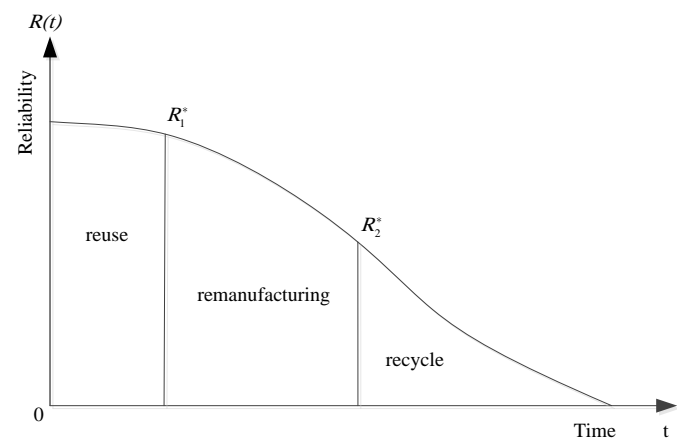

Figure 2. Illustration of the proposed decision-making model for the remanufacturing scheme of a given component.

(1) When $R(t)>R_{1}^{*}$, the component reliability is in the safe area. In this case, the used components can be reused directly without remanufacturing or additional processing. This case is referred to as reuse.

(2) For $R_{2}^{*}<R(t)<R_{1}^{*}$, the components may not have failed yet. However, there exist potential failure points and the probability of failure occurring during this period of time is larger. Therefore, the components should be remanufactured to improve their reliability. This case is referred to as remanufacturing.

(3) When $R(t)<R_{2}^{*}$, the components should not be suggested for a remanufacturing due to the required processing costs and the technical feasibility. The best disposal method is to recycle and reclaim useful materials in order to save resources.

\section{Case study}

The transfer case is the switching mechanism of the concrete pump truck between the driving and pump sending positions. The bearing on the transfer case is the key component prone to failure and restricts the service life of the transfer case. The bearing adds a very high additional value to the machine and has a very good effect in terms of energy saving and material saving. The selection of the appropriate remanufacturing plan directly affects the effective utilization of the additional value of the bearing. Here, the maintenance-mode decision for the bearing on the transfer case was used as a case study to validate the above established model.

The evaluation of the reliability of the bearing was based on the historical life data of the bearing, given in Table 1, which was empirically determined by a certain company.

Table 1. The historical life data of the bearing

\begin{tabular}{ccc||ccc||ccc}
\hline No. & Working hours & State & No. & Working hours & State & No. & Working hours & State \\
\hline 1 & $1892 \mathrm{~h}$ & Failure & 11 & $5187 \mathrm{~h}$ & Failure & 21 & $8648 \mathrm{~h}$ & Pause \\
2 & $2712 \mathrm{~h}$ & Failure & 12 & $5404 \mathrm{~h}$ & Failure & 22 & $9012 \mathrm{~h}$ & Failure \\
3 & $2840 \mathrm{~h}$ & Pause & 13 & $5536 \mathrm{~h}$ & Pause & 23 & $9392 \mathrm{~h}$ & Failure \\
4 & $2885 \mathrm{~h}$ & Pause & 14 & $5774 \mathrm{~h}$ & Failure & 24 & $9748 \mathrm{~h}$ & Failure \\
5 & $3092 \mathrm{~h}$ & Failure & 15 & $6016 \mathrm{~h}$ & Failure & 25 & $10322 \mathrm{~h}$ & Pause \\
6 & $4078 \mathrm{~h}$ & Failure & 16 & $6472 \mathrm{~h}$ & Pause & 26 & $10506 \mathrm{~h}$ & Pause \\
7 & $4204 \mathrm{~h}$ & Failure & 17 & $6680 \mathrm{~h}$ & Failure & 27 & $11049 \mathrm{~h}$ & Failure \\
8 & $4456 \mathrm{~h}$ & Failure & 18 & $6812 \mathrm{~h}$ & Failure & 28 & $12742 \mathrm{~h}$ & Failure \\
9 & $4738 \mathrm{~h}$ & Failure & 19 & $6984 \mathrm{~h}$ & Pause & & & \\
10 & $5113 \mathrm{~h}$ & Pause & 20 & $8480 \mathrm{~h}$ & Pause & & & \\
\hline
\end{tabular}

Note: The data with the state "Failure" corresponds to cases where the bearing was replaced due to failure 
during the data acquisition period and the data with the state "Pause" corresponds to cases where the bearing was replaced not due to failure during the data acquisition period.

The data in Table 1 includes both failure data and pause data. By removing the pause data and ranking the failure data from small to large again, the three-parameter Weibull distribution model can be established and the values obtained after removing the pause data are listed in Table 2.

Table 2. The failure data obtained for the bearing after elimination of the non-failure data

\begin{tabular}{ccc||ccc}
\hline No. & Working hours & State & No. & Working hours & State \\
\hline 1 & $1892 \mathrm{~h}$ & Pause & 10 & $5774 \mathrm{~h}$ & Pause \\
2 & $2712 \mathrm{~h}$ & Pause & 11 & $6016 \mathrm{~h}$ & Pause \\
3 & $3092 \mathrm{~h}$ & Pause & 12 & $6680 \mathrm{~h}$ & Pause \\
4 & $4078 \mathrm{~h}$ & Pause & 13 & $6812 \mathrm{~h}$ & Pause \\
5 & $4204 \mathrm{~h}$ & Pause & 14 & $9012 \mathrm{~h}$ & Pause \\
6 & $4456 \mathrm{~h}$ & Pause & 15 & $9392 \mathrm{~h}$ & Pause \\
7 & $4738 \mathrm{~h}$ & Pause & 16 & $9748 \mathrm{~h}$ & Pause \\
8 & $5187 \mathrm{~h}$ & Pause & 17 & $11049 \mathrm{~h}$ & Pause \\
9 & $5404 \mathrm{~h}$ & Pause & 18 & $12742 \mathrm{~h}$ & Pause \\
\hline
\end{tabular}

The least square method, the correlation coefficient method and the mean rank order method were used to estimate the three parameters of the Weibull distribution. The position parameter $t_{0}$ was determined first and its initial value $t_{01}$ is the minimum service life according to the experimental data presented in Table 2. Then, the three-parameter Weibull distribution was regressed linearly by parameter transformation and the correlation coefficient $\rho_{1}$ was computed. Then, the iterations were started according to $t_{02}=t_{01}-0.02 t_{01}$, and the correlation coefficient $\rho_{\mathrm{i}}$ for each $t_{0 i}$ was calculated. For $t_{0}=795$, the maximum correlation coefficient was determined to be 0.9831 . Then, the remaining two parameters of the Weibull distribution model were computed to $\beta=1.90$ and $\eta=7875$ using the least square and mean rank order methods.

The degree of reliability of the bearing can be obtained by substituting the above three parameter values into Eq. (3): $R(t)=\exp \left[-\left(\frac{t-795}{7875}\right)^{1.9}\right]$

Supposed a bearing has failed after 5000 hours of operation. The degree of reliability of the bearing after 5000 hours of operation can be calculated by substituting the three parameters $\beta, \eta, t_{0}$ into Eq. (3): $R(5000)=\exp \left[-\left(\frac{5000-795}{7875}\right)^{1.9}\right]=0.74$

According to the threshold values provided by the manufacturer, i.e., $R_{1}^{*}=0.8$ and $R_{2}^{*}=0.48$, the degree of reliability of the bearing after failure of 0.74 is between the reliability threshold values $R_{1}^{*}$ and $R_{2}^{*}$, and hence the bearing should be remanufactured. Up to now, the company which provided the empirical data always recycles the bearings, which results in a waste of resources. However, if the appropriate remanufacturing scheme is adopted according to the reliability evaluation of the bearing, such a situation can be avoided.

\section{Conclusions}

(1) Compared with the two-parameter Weibull distribution, the three-parameter Weibull distribution function is more complex when used for evaluating the reliability of components. However, it is more appropriate for the actual situation and is more precise.

(2) The model was applied to the remanufacturing scheme decision-making process for the 
bearing on the transfer case of a concrete pump truck, and the results have shown that the proposed model can better reflect the relationship between the reliability of the bearing and the desired remanufacturing scheme. This study can provide technical support for the research on remanufacturing scheme decision-making models and active remanufacturing technology of the used components.

In addition to the reliability of the used components, other factors influencing the decision for remanufacturing scheme include technological, economic, and environmental concerns. These factors, as well as the feasibility of the remanufacturing of the used components will be comprehensively considered in further studies to realize a maximization of the value of the remanufacturing value.

\section{References}

[1] Xu Binshi, State of the art and future development in remanufacturing engineering. Transactions of Materials and Heat Treatment, . 31(1), 2007, 10-14.

[2] A. Rashid, F. M. A. Asif, P. Krajnik, and C. M. Nicolescu, "Resource conservative manufacturing: an essential change in business and technology paradigm for sustainable manufacturing," Journal of Cleaner Production,vol.57(15), 2013,p.166-177.

[2] Gonzalez-torre B, Adenso-diaz B, Optimizing decision making at the end of life of a product. Proceedings of the SPIE-The International Society for Optical Engineering, Vol. 52 (1),2003, p.40-50.

[3]Ghazalli Z, Murata A, Development of an AHP-CBR evaluation system for remanufacturing: End-of-life selection strategy. International Journal of Sustainable Engineering, Vol. 4(1) 2011, p.2-15.

[4] Ghazalli Z, Murata A, An integrated TSP - GAwith EOL cost model for selecting the best EOL option. International Journal of Industrial Engineering Computations, Vol. 2(4), 2011, p.775792.

[5] Zeng Shoujin, Jiang Jibin, Xu Mingsan, Research on assessment model of electromechanical products remanufacturability. Journal of Fujian University of Technology, Vol.7(3), 2009, p.271-274.

[6] Du Yanbin, Cao nuajun, Liu Fei, et a1. Evaluation of machine tool remanufacturing scheme based on entropy weight and AI-IP. Computer Integrated Manufacturing Systems, Vol.17(1), 2011, p.84-88.

[8] G. Liu, T. Liu, Q.D Ke., S.X Song., D. Zhou, “Time interval decision-making methods for active remanufacturing product based on game theory and neural network" , Journal of Mechanical Engineering, vol. 49(7), 2013, p. 29-35.

[9] Ling Dan. Weibull distribution model and its applicationresearch on the mechanical reliability, edtied by University of electronic science and technology, 2010

[10] Yu Xiaohong, Zhang Laibin, Wang Zhaohui, Duan Lixiang, Reliability life analysis of the equipment based on new weibull distribution parameter estimation method. Journal of Mechanical Strength, Vol. 29(2007), p. 932-936. 